\title{
Anemia e Transfusões de Concentrados de Hemácias em Pacientes Graves nas UTI Brasileiras (pelo FUNDO-AMIB)*
}

\section{Transfusion Practices in Brazilian Intensive Care Units (pelo FUNDO-AMIB)}

\author{
Suzana Margareth Lobo'; Silvia Rios Vieira², Marcos Freitas Knibel; Cintia Magalhães Carvalho Grion"; \\ Gilberto Friedman'; Jorge Luis Valiatti6; Flávia Ribeiro Machado7; Paulo Antonio Chiavone;; \\ Luis Eduardo Miranda Paciência ${ }^{9}$; Juarez de Paula ${ }^{10}$; Sérgio Mussi Guimarães ${ }^{11}$; João Luiz Ferreira Costa ${ }^{12}$; \\ Rubens Carmo Costa Filho ${ }^{13}$; Gleida Alves Borges ${ }^{14}$; Hemerson Casado Gama ${ }^{16}$; Marcellus Gazola Grilo ${ }^{17}$; \\ Kerginaldo Paulo Torres ${ }^{18}$; Rubens Sérgio da Silva Franco ${ }^{19}$; Jorge Eduardo Silva Soares Pinto ${ }^{20}$; Cid Marcos David ${ }^{21}$
}

\section{RESUMO}

JUSTIFICATIVA E OBJETIVOS: A anemia é uma condição comum em pacientes graves. A transfusão de hemoderivados aumenta de forma significativa o risco de transmissão de agentes infecciosos e afeta o perfil imunológico. O objetivo deste estudo foi avaliar a incidência de anemia e a prática de transfusão de hemá- cias em UTI brasileiras.

MÉTODO: Estudo prospectivo, multicêntrico, realizado em 19 UTI em um período de duas semanas. A presença de anemia, as indicações e a utilização de concentrados de hemácias, foram avaliadas diariamente. As complicações que ocorreram durante a internação na UTI e após a transfusão da primeira unidade de concentrado de hemácias foram registradas.
1. Professora Adjunta de Clinica Médica da Faculdade de Medicina de São José do Rio Preto - SP, Coordenadora do Serviço de Terapia Intensiva e da Residência Medica em Medicina Intensiva. Doutora em Medicina pela Universidade de São Paulo.

2. Professora Adjunta, Departamento de Medicina Interna, FM/UFRGS, Serviço de Medicina Intensiva, HC de Porto Alegre, RS, Brasil. Doutora em Medicina pela UFRGS, Porto Alegre, RS, Brasil. PósDoutora em Medicina pela Universidade Paris VI, França.

3. Mestre em Terapia Intensiva-UFRJ, Chefe dos CTI dos Hospitais Cardiotrauma Ipanema e São Lucas, RJ - Título de Especialista pela AMIB

4. Doutora em Medicina e Ciência da Saúde - Universidade Estadual de Londrina (UEL), Mestre em Medicina Interna da UEL, Especialista em Medicina Intensiva - AMIB

5. Professor Adjunto de Medicina Interna, FAMED-UFRGS, Serviço de Medicina Intensiva do HCPA-UTI Central do Complexo Hospitalar Santa Casa, Coordenador do Programa de Residência Médica, Editor da RBTI

6. Professor Adjunto de Clínica Médica da Faculdade de Medicina de Catanduva. Mestre e Doutor pela UNIFESP-EPM. Médico Intensivista- Supervisor das UTI do Complexo Hospitalar da Fundação Padre Albino, Catanduva, SP

7. Professora Adjunta e Chefe do Setor de Terapia da Disciplina de Anestesiologia, Dor e Terapia Intensiva da Universidade Federal de São Paulo

8. Professor Assistente da FCMSCSP, Doutor e Mestre em Medicina pela FCMSCSP, Diretor do Serviço de Terapia Intensiva da Santa Casa de Misericórdia de São Paulo

9. Especialista em medicina intensiva pela AMIB. Coordenador da Unidade de Terapia Intensiva do Hospital Unimed de Limeira 10. Especialista pela AMIB, Especialista Sociedade Brasileira de Clínica Médica, Coordenador da UTI do Hospital Regional de Assis, SP 12. Professor Assistente do Departamento de Medicina - Serviço de Terapia Intensiva, Chefe do Serviço de Nutrição Parenteral e Enteral, Especialista em Medicina Intensiva pela AMIB
13. Fellow of American College of Chest Physician. MBA de Marketing Pela Fundação Getúlio Vargas, RJ. Chefe das Terapias Intensivas do Hospital Pró Cardíaco

13. Coordenador da Rotina da Terapia Intensiva do Hospital Pro Cardíaco. Professor Auxiliar de Clinica Médica da UNIRIO. Coordenador do Centro do Rim do Hospital Pro Cardíaco. MBA de Administração pela FGV, RJ. MBA de Saúde pela FGV, RJ

14. Especialista em medicina intensiva pela AMIB. Coordenadora da Unidade de Terapia Intensiva do Hospital Santa Helena de Goiânia, GO.

15. Especialista em Cirurgia Cardiovascular pela Sociedade Brasileira de Cirurgia Cardiovascular; Coodenador da Unidade coronariana do Hospital evangélico de Cachoeiro de Itapemirim, ES.

16. Título de Especialista em Nefrologia pela SBN, Título de Especialista em Medicina Intensiva pela AMIB, Chefe UTI Adulto e Coronariana Hospital Evangélico Cachoeiro de Itapemirim, ES

17. Mestre em Farmacologia Cardiovascular UFRJ, Professor Adjunto de Farmacologia Cardiovascular da UFRN, Coordenador da UTI do Hospital Onofre Lopes da UFRN, Rio de Janeiro, RJ

18. Especialista em Medicina Intensiva pela AMIB. Coordenador da Unidade de Terapia Intensiva do Hospital Novo Atibaia, Atibaia, SP 19. Especialista em Medicina Intensiva pela AMIB. Membro do Fundo AMIB.

20. Especialista em medicina intensiva pela AMIB. Doutor em Ciências Médicas

pela UFRJ. Coordenador da Divisão de Pesquisa do Fundo AMIB.

Apresentado em 04 de junho de 2006

Aceito para publicação em 04 de setembro de 2006

Endereço para correspondência:

Avenida Brigadeiro Faria Lima, 5544 - Hospital de Base $7^{\circ}$ Andar 15090-000 São José do Rio Preto, SP

E-mail: utigeral.hbase@famerp.br

(C)Associação de Medicina Intensiva Brasileira, 2006 
RESULTADOS: Um total de 33\% apresentava anemia na admissão na UTI e esta proporção aumentou para $55 \%$ no final de sete dias de internação. Um total de 348 unidades de concentrado de hemácias foi transfundido em 86 pacientes (36,5\%). A média de suas unidades por paciente foi $4,1 \pm 3,3 \mathrm{U}$. O nível de hemoglobina limiar para a transfusão de $\mathrm{CH}$ foi $7,7 \pm 1,1$ $\mathrm{g} / \mathrm{dL}$. Pacientes transfundidos tinham mais disfunções orgânicas avaliadas pelo escore SOFA $(7,9 \pm 4,6$ versus $5,6 \pm 3,8$, transfundidos versus não transfundidos, $p<$ $0,05)$. As taxas de mortalidade foram $43,5 \%$ e $36,3 \%$ em pacientes transfundidos e não transfundidos, respectivamente (RR 0,61-11,7, NS). Pacientes transfundidos tiveram número maior de complicações (1,58 \pm 0,66 versus $1,33 \pm 0,49, p=0,0001$ ).

CONCLUSÕES: A anemia é comum em UTI brasileiras. O limiar transfusional de hemoglobina foi menor do que o observado em outros paises.

Unitermos: Anemia, paciente grave, transfusão de hemácias

\section{SUMMARY}

BACKGROUND AND OBJECTIVES: Anemia of critical illness is a multifactorial condition caused by blood loss, frequent phlebotomies and inadequate production of red blood cells (RBC). Controversy surrounds the most appropriate hemoglobin concentration "trigger" for transfusion of RBC. We aimed to evaluate transfusion practices in Brazilian ICUs.

METHODS: A prospective study throughout a 2-week period in 19 Brazilian ICUs. Hemoglobin $(\mathrm{Hb})$ level, transfusion rate, organ dysfunction assessment and 28-day mortality were evaluated. Primary indication for transfusion and pretransfusion hemoglobin level were collected for each transfusion.

RESULTS: Two hundred thirty-one patients with an ICU length of stay longer than $48 \mathrm{~h}$ were included. An $\mathrm{Hb}$ level lower than $10 \mathrm{~g} / \mathrm{dL}$ was found in $33 \%$ on admission in the ICU. A total of 348 RBC units were transfused in 86 patients (36.5\%). The mean pretransfusion hemoglobin level was $7.7 \pm 1.1 \mathrm{~g} / \mathrm{dL}$. Transfused-patients had significantly higher SOFA score $(7.9 \pm 4.6$ vs 5.6 $\pm 3.8, p<0.05$, respectively), days on mechanical ventilation $(10.7 \pm 8.2$ vs $7.2 \pm 6.4, p<0.05)$ and days on vasoactive drugs $(6.7 \pm 6.4$ vs $4.2 \pm 4.0, p<0.05)$ than non-transfused patients despite similar APACHE II scores $(15.2 \pm 8.1$ vs $14.2 \pm 8.1$, NS). Transfused patients had higher mortality rate $(43.5 \%)$ than non-transfused patients (36.3\%) (RR 0.60-1.15, NS). Only one patient
$(0.28 \%)$ had febrile non-hemolytic transfusion and urticarial reactions.

CONCLUSIONS: Anemia is common in critically ill patients. It seems from the present study that transfusion practices in Brazil have had a more restrictive approach with a lower limit "transfusion trigger".

Key Words: anemia, blood transfusion, critically ill patients, red cells

\section{INTRODUÇÃO}

A anemia é uma condição de ocorrência comum em pacientes graves ${ }^{1}$. Em geral, ocorre em conseqüência de uma associação de fatores, como a diluição secundária à reposição volêmica, a hemólise, as anormalidades no metabolismo do ferro, as perdas sangüíneas pelo trato gastrintestinal e, ainda, com a diminuição da produção de eritropoetina com conseqüente diminuição de eritropoese em resposta a presença de citocinas inflamatórias ${ }^{1-6}$. As flebotomias freqüentes para análises sangüíneas são responsáveis por cerca de $40 \mathrm{~mL} /$ dia de perda sangüínea ${ }^{7}$. O fator de necrose tumoral e a interleucina-1 (IL-1) inibem o crescimento das células pró-eritrogênicas na medula óssea em pacientes com a síndrome da resposta inflamatória sistêmica ${ }^{4}$. Aproximadamente $40 \%$ a $50 \%$ dos pacientes admitidos em Unidades de Terapia Intensiva (UTI) recebem no mínimo uma transfusão de hemácias com uma média de cinco unidades por paciente ${ }^{5}$.

A oferta global de oxigênio é determinada apenas pelo débito cardíaco e pelo conteúdo sangüíneo de oxigênio. O principal determinante do conteúdo sangüíneo de oxigênio é a saturação da hemoglobina. Ela é a única forma natural de transportar oxigênio em quantidades significativas. Dessa forma, existe base racional para administração de concentrado de hemácias como forma de melhorar a oxigenação tecidual. Contudo, com exceção do período inicial do choque e da situação de dependência do consumo de oxigênio em relação à oferta, a administração de concentrado de hemácias não produz melhora no consumo de oxigênio ${ }^{8}$. Além disso, a transfusão de hemoderivados aumenta de forma significativa o risco de transmissão de agentes infecciosos e afeta o perfil imunológico, aumentando o risco de infecção nosocomial e de recorrência de câncerr $^{9-13}$.

O nível mínimo de hemoglobina tolerado em indivíduos saudáveis ou doentes sem efeitos adversos é desconhecido ${ }^{14}$. Estudo recente mostrou que um nível de hemoglobina de $7 \mathrm{~g} / \mathrm{dL}$ pode ser adequado para a maioria 
dos pacientes ${ }^{15}$. Embora a presença de anemia tenha sido relacionada com maior probabilidade de óbito, a correção da anemia por meio de transfusão parece não determinar a diminuição do risco. Ao contrário, a administração de concentrado de hemácias é fator independente para morbidade e mortalidade em pacientes internados nas UTI ${ }^{13,16}$. Embora os dados de um estudo retrospectivo tenham previamente demonstrado que a transfusão de hemácias seria benéfica em pacientes idosos e com infarto agudo do miocárdio, a análise de uma grande população de pacientes com síndromes coronarianas agudas demonstrou que, mesmo neste subgrupo, ela associa-se à maior risco de morte ou de evento cardíaco ${ }^{17,18}$.

As práticas transfusionais variam significativamente entre diferentes países e há relatos de que a proporção de pacientes transfundidos pode atingir $85 \%$, quando o tempo de permanência na UTI ultrapassa uma semana $^{19,20}$.

O objetivo deste estudo foi avaliar a incidência de anemia e a prática de transfusão de concentrado de hemácias em UTI brasileiras, assim como a sua correlação com complicações e com mortalidade.

\section{MÉTODO}

Após aprovação pelo Comitê de Ética em pesquisa da instituição coordenadora, foi realizado um estudo prospectivo, multicêntrico, observacional, realizado em 19 UTI (sete hospitais universitários, oito hospitais privados, dois hospitais públicos e dois públicos-privados) de seis Estados da Federação, em um período de duas semanas (22 de novembro a 6 de dezembro de 2004). O termo de consentimento livre e esclarecido foi dispensado. Foram incluídos os pacientes com mais de 18 anos e com permanência em UTI clínica ou cirúrgica por mais de 48 horas. Os pacientes foram acompanhados por 28 dias. Os critérios de exclusão foram insuficiência renal crônica, cirrose hepática, mielodisplasias ou pacientes testemunhas de Jeová.

A anemia foi considerada na presença de um nível de $\mathrm{Hb}<10 \mathrm{~g} / \mathrm{L}$. Avaliou-se a dosagem sérica da hemoglobina, obtida na rotina de coleta de sangue da manhã. O valor que desencadeou a transfusão de concentrado de hemácias foi considerado o pré-transfusional. As razões para a transfusão de hemácias foram classificadas de acordo com as seguintes categorias: baixo nível de hemoglobina, presença de sangramento ativo ou de sinais de isquemia miocárdica para melhorar a oferta de oxigênio tecidual, procedimento cirúrgico e instabilidade hemodinâmica. Registraram-se o uso de ventilação mecânica invasiva e de fármacos vasoativos (dopamina $>5 \mu \mathrm{g} / \mathrm{kg} / \mathrm{h}$ ou noradrenalina, qualquer dose). As complicações que ocorreram durante a internação na UTI e após a transfusão da primeira unidade de concentrado de hemácias foram registradas. Infecção nosocomial foi considerada de acordo com parâmetros clínicos, laboratoriais e microbiológicos usuais. Sepse e choque séptico foram definidos de acordo com os critérios da ACCP/SCCM ${ }^{21}$. Disfunções orgânicas foram avaliadas com o uso do escore SOFA Seqüencial Organ Failure Assessment ${ }^{22}$. A síndrome de angústia respiratória aguda (SARA) foi definida na presença de infiltrados pulmonares bilaterais na radiografia de tórax com pressão capilar da artéria pulmonar $<18 \mathrm{mmHg}$ ou na ausência de evidência clínica de hipertensão atrial esquerda, e da razão entre a pressão parcial arterial de oxigênio $\left(\mathrm{PaO}_{2}\right)$ e a fração inspirada de oxigênio $\left(\mathrm{FiO}_{2}\right)<200$. Edema pulmonar foi considerado na presença de sinais radiológicos de edema pulmonar em associação a pressão de oclusão da artéria pulmonar $>18 \mathrm{mmHg}$ ou aos sinais de disfunção miocárdica no ecocardiograma. Uma reação transfusional foi considerada na presença de febre, calafrios, urticária ou reação hemolítica que ocorreram em conseqüência de uma transfusão de hemácias.

Considerando-se uma proporção de casos de anemia de 30\% nas UTI brasileiras, seria necessária a inclusão de 224 a 323 pacientes no estudo para uma precisão de estimativa de $5 \%$ a $6 \%$. Na análise univariada, foi usado os testes $t$ de Student para as variáveis contínuas e o Exato de Fisher para as variáveis qualitativas. A sobrevida foi estimada pelo método de Kaplan-Meyer e para a comparação entre os grupos foi usado o teste de log-rank. Para todos os testes o valor de $p<0,05$ foi considerado estatisticamente significativo. $\mathrm{Na}$ análise foi usado o minitab statistical software.

\section{RESULTADOS}

Os dados demográficos e clínicos dos pacientes estão demonstrados na tabela 1 . Um total de $59,3 \%$ dos pacientes estava sob ventilação mecânica e 40,6\% receberam fármaco vasoativo. Na admissão na UTI, 33\% apresentavam anemia. No final da primeira semana de internação, a proporção de pacientes com anemia aumentou para $55 \%$. A tabela 2 mostra a correlação entre diagnósticos de admissão na UTI com a necessidade de transfusão e a quantidade de concentrados de hemácias. A figura 1 demonstra os níveis médios 
de hemoglobina durante a internação. Os seus níveis diminuem progressivamente após a admissão e atingem o nível mais baixo no $21^{\circ}$ dia de internação $(9,24$ $\pm 1,3 \mathrm{~g} / \mathrm{dL})$.

Tabela 1 - Dados Demográficos, Doenças de Base e Dados Clínicos dos Pacientes.

\begin{tabular}{|c|c|}
\hline Número de pacientes & 231 \\
\hline Idade (anos) & $60,8 \pm 18,3$ \\
\hline Sexo $(M / F)$ & $54,3 \% / 45,7 \%$ \\
\hline APACHE II escore & $14,6 \pm 8,1$ \\
\hline SOFA escore, admissão & $6,5 \pm 4,3$ \\
\hline Hipertensão arterial & $44 \%$ \\
\hline Diabete & $19 \%$ \\
\hline Doença pulmonar obstrutiva crônica & $11 \%$ \\
\hline Neoplasia & $9 \%$ \\
\hline Doença do trato gastrintestinal & $5 \%$ \\
\hline Insuficiência renal aguda & $4 \%$ \\
\hline Pacientes com anemia na admissão & $76(33 \%)$ \\
\hline Pacientes com anemia na primeira semana & $127(55 \%)$ \\
\hline Pacientes transfundidos & $86(36,5 \%)$ \\
\hline $\begin{array}{l}\text { Total de concentrados de hemácias transfun- } \\
\text { didas (U) }\end{array}$ & 348 \\
\hline $\begin{array}{l}\text { Total de concentrado de hemácias transfun- } \\
\text { didas por paciente }(U)\end{array}$ & $4,1 \pm 3,3$ \\
\hline Hemoglobina na admissão (g/dL) & $11,4 \pm 2,6$ \\
\hline Hemoglobina pré-transfusional (g/dL) & $7,7 \pm 1,1$ \\
\hline Tempo UTI, dias (mediana, $25^{\circ}$ e $75^{\circ}$ percentil) & $6(4-7)$ \\
\hline $\begin{array}{l}\text { Tempo hospital, dias (mediana, } 25^{\circ} \text { e } 75^{\circ} \text { per- } \\
\text { centil) }\end{array}$ & $14(7-27)$ \\
\hline Ventilação mecânica (\%) & $59,3 \%$ \\
\hline Tempo de ventilação mecânica (dias) & $8,9 \pm 7,5$ \\
\hline Fármacos vasoativos (\%) & $40,6 \%$ \\
\hline Tempo DVA (dias) & $5,7 \pm 5,7$ \\
\hline Mortalidade (28 dias) (\%) & $38,9 \%$ \\
\hline
\end{tabular}

APACHE: Acute Physiology and Chronic Health Evaluation; SOFA, Sequential Organ Failure Assessment.

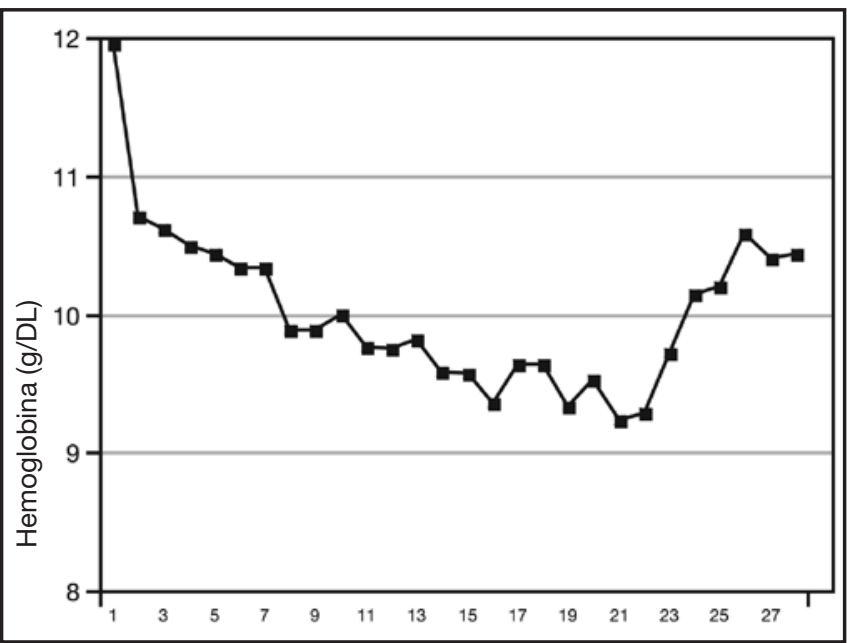

Figura 1 - Concentrações Médias de Hemoglobina durante a Internação (28 dias).

Um total de 348 unidades de concentrado de hemácias $(\mathrm{CH})$ foi transfundido em 86 pacientes (36,5\%). Do total de $\mathrm{CH}$ utilizados $25 \%$ foram administrados em pacientes no pós-operatório, $15 \%$ em pacientes com insuficiência renal aguda e $12 \%$ em pacientes politraumatizados (Tabela 2). A taxa média da hemoglobina basal foi $11,4 \pm 2,6 \mathrm{~g} / \mathrm{dL}$. A média de unidades de $\mathrm{CH}$ por paciente foi 4,1 $\pm 3,3 \mathrm{U}$. O nível de hemoglobina limiar para a transfusão de $\mathrm{CH}$ foi $7,7 \pm 1,1 \mathrm{~g} / \mathrm{dL}$. Os pacientes portadores de coronariopatias (23) tiveram um nível médio de hemoglobina pré-transfusional de $8 \pm 1,3 \mathrm{~g} / \mathrm{dL}$. As razões para transfusão de hemácias estão listadas na tabela 3 . As principais razões foram baixo nível de hemoglobina (53\%) e sangramento ativo (26.6\%). A figura 2 representa a distribuição das transfusões durante a internação. A maior taxa de transfusão de hemácias ocorreu na primeira semana.

Tabela 2 - Distribuição dos Diagnósticos de Admissão e Correlação com a Necessidade de Transfusão e Quantidade de Unidades de Concentrados de Hemácias.

\begin{tabular}{lccc}
\hline Causa da Admissão & $\mathrm{N}^{\circ}$ de Pacientes (\%) & Transfundidos (\%) & $\mathrm{N}^{\circ}$ de Unidades \\
\hline Pós-operatório & $73(31,6)$ & $27(37 \%)$ & $87(25 \%)$ \\
Insuficiência respiratória aguda & $33(14,2)$ & $11(33 \%)$ & $57(16 \%)$ \\
Sepse & $19(8,2)$ & $10(53 \%)$ & $22(6,3 \%)$ \\
Trauma & $13(5,6)$ & $10(77 \%)$ & $42(12 \%)$ \\
Coronariopatia & $12(5,2 \%)$ & $1(7,7 \%)$ & $12(3,4 \%)$ \\
Insuficiência renal aguda & $11(4,8 \%)$ & $7(64 \%)$ & $53(15 \%)$ \\
Insuficiência cardíaca & $10(4,3 \%)$ & $4(40 \%)$ & $6(1,7 \%)$ \\
Pneumonia & $9(3,9 \%)$ & $4(44 \%)$ & $6(1,7 \%)$ \\
Doença pulmonar obstrutiva crônica & $6(2,6 \%)$ & $1(17 \%)$ & $6(1,7 \%)$ \\
Choque hemorrágico & $4(1,7 \%)$ & $4(100 \%)$ & $14(4,0 \%)$ \\
Hemorragia digestiva & $2(0,8 \%)$ & $2(100 \%)$ & $17(4,9 \%)$
\end{tabular}


Tabela 3 - Causas de Transfusão de Hemácias.

\begin{tabular}{lc}
\hline Nível de hemoglobina & $53 \%$ \\
Sangramento ativo & $26,6 \%$ \\
Sinais de isquemia tecidual & $9,9 \%$ \\
Aumento do transporte de oxigênio & $7,3 \%$ \\
Procedimento cirúrgico & $4,1 \%$ \\
Instabilidade hemodinâmica & $2,3 \%$ \\
Outras & $0,8 \%$ \\
\hline
\end{tabular}

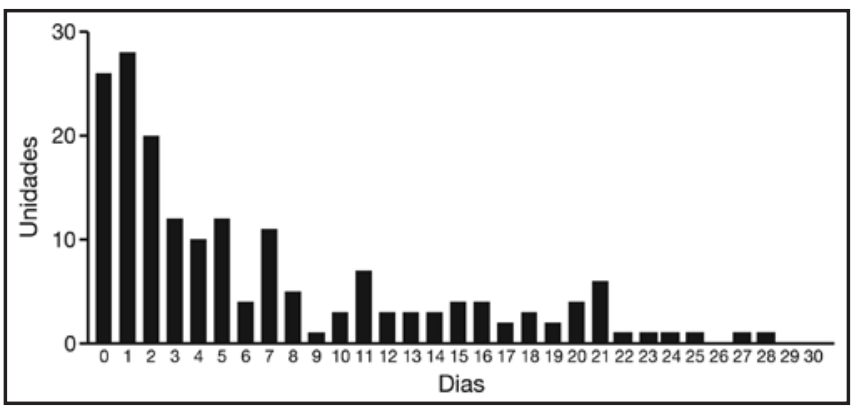

Figura 2 - Distribuição das Transfusões de Hemácias (unidades) durante 28 Dias.

Pacientes transfundidos tiveram taxas de hemoglobina na admissão da UTI significativamente menores $(9,9 \pm 2,5 \mathrm{~g} / \mathrm{dL})$ do que os não transfundidos (12,2 \pm $2,3 \mathrm{mg} / \mathrm{dL})(\mathrm{p}<0,05)$ (Tabela 4). Pacientes transfundidos receberam ventilação mecânica mais freqüentemente $(78,8 \%)$ em comparação aos não transfundidos $(47,9 \%)(p<0,05)$. O mesmo ocorreu com a freqüência de uso de fármacos vasoativos $(66,6 \%$ versus $25,8 \%$, $p<0,05)$. Embora com valores de APACHE II semeIhante na admissão, os pacientes transfundidos tinham mais disfunções orgânicas avaliadas pelo escore SOFA
$(7,9 \pm 4,6$ versus $5,6 \pm 3,8$, transfundidos versus não transfundidos, $\mathrm{p}<0,05)$. As taxas de mortalidade foram $43,5 \%$ e $36,3 \%$ em pacientes transfundidos e não transfundidos, respectivamente (RR 0,61-11,7, NS). As curvas de sobrevida de pacientes transfundidos e não transfundidos (Kaplan-Meyer) estão representadas na figura 3.

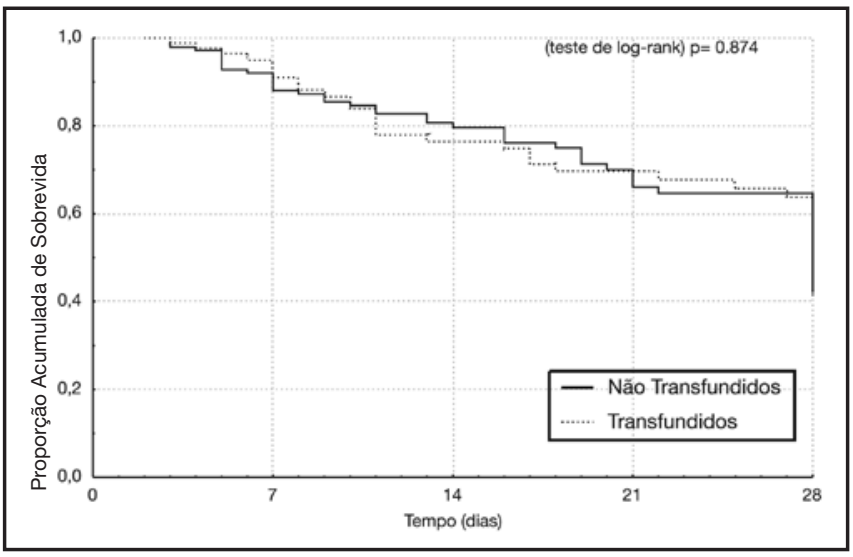

Figura 3 - Curva de Sobrevida de Pacientes Transfundidos e não Transfundidos.

Um total de $14,1 \%$ dos pacientes transfundidos apresentou complicações em comparação a 7,6\% no grupo de pacientes não transfundidos (RR 1,86, IC 95\% 0,87 $-3,95$, NS) (Tabela 5). Pacientes transfundidos tiveram número maior de complicações $(1,58 \pm 0,66$ vs $1,33 \pm$ $0,49, p=0,0001)$. Uma reação adversa relacionada à transfusão de hemácias foi relatada em somente um paciente $(0,28 \%)$, que apresentou uma reação febril e urticariforme.

Tabela 4 - Pacientes Transfundidos e não Transfundidos

\begin{tabular}{|c|c|c|c|}
\hline Variáveis & $\begin{array}{c}\text { Transfundidos } \\
(n=85)\end{array}$ & $\begin{array}{c}\text { Não Transfundidos } \\
(n=146)\end{array}$ & $p$ \\
\hline Idade (anos) & $60,2 \pm 20,4$ & $61,2 \pm 17,0$ & 0,686 \\
\hline Sexo masculino & $39(46,4 \%)$ & $86(58,9 \%)$ & 0,074 \\
\hline APACHE II & $15,2 \pm 8,1$ & $14,2 \pm 8,1$ & 0,364 \\
\hline SOFA na admissão & $7,9 \pm 4,6$ & $5,6 \pm 3,8$ & $<0,001$ \\
\hline Hemoglobina na admissão (g/dL) & $9,9 \pm 2,5$ & $12,2 \pm 2,3$ & $<0,001$ \\
\hline Ventilação mecânica & $67(78,8 \%)$ & $70(47,9 \%)$ & $<0,001$ \\
\hline Tempo de ventilação mecânica (dias) & $10,7 \pm 8,2$ & $7,2 \pm 6,4$ & 0,006 \\
\hline Vasopressores & $56(66,6 \%)$ & $38(25,8 \%)$ & $<0,001$ \\
\hline Tempo de uso de vasopressores (dias) & $6,7 \pm 6,4$ & $4,2 \pm 4,0$ & 0,024 \\
\hline Taxa de mortalidade (28 dias) & $37(43,5 \%)$ & $53(36,3 \%)$ & 0,327 \\
\hline
\end{tabular}

Análise Univariada

APACHE: Acute Physiology and Chronic Health Evaluation; SOFA: Sequential Organ Failure Assessment. 
Tabela 5 - Complicações em Pacientes Transfundidos e não Transfundidos.

\begin{tabular}{lcc}
\hline & $\begin{array}{c}\text { Transfundidos } \\
(\mathrm{n}=85)\end{array}$ & $\begin{array}{c}\text { Não Transfundidos } \\
(\mathrm{n}=146)\end{array}$ \\
\hline $\begin{array}{l}\text { Pacientes com complica- } \\
\text { ções }\end{array}$ & $12(14,1 \%)$ & $12(7,6 \%)$ \\
$\begin{array}{l}\text { Complicações por pa- } \\
\text { ciente }\end{array}$ & $1,58 / 0,66$ & $1.33 / 0.49^{*}$ \\
$\quad$ SDRA & $2(2,3 \%)$ & $1(0,7 \%)$ \\
Edema agudo de pul- & $1(1,2 \%)$ & $1(0,7 \%)$ \\
mão & $9(10,6 \%)$ & $9(6,3 \%)$ \\
Infecção nosocomial & $1(1,2 \%)$ & $0(0 \%)$ \\
Sepse & $2(2,3 \%)$ & $3(2,1 \%)$ \\
Choque séptico & $3(3,6 \%)$ & $1(0,7 \%)$ \\
Hemorragia digestiva & $0(0 \%)$ & $1(0,7 \%)$ \\
Tromboembolismo pul- \\
monar
\end{tabular}

SDRA: Síndrome do Desconforto Respiratório Agudo.

* 0,05 versus transfundidos.

\section{DISCUSSÃo}

A anemia é comum em UTI brasileiras. Na admissão na UTI, 33\% dos pacientes apresentavam anemia. No final da primeira semana, 55\% apresentavam anemia. Um total de $36,5 \%$ dos pacientes internados recebeu transfusão. O nível de hemoglobina que desencadeou a transfusão de $\mathrm{CH}$ foi $7,7 \pm 1,1 \mathrm{~g} / \mathrm{dL}$. Os pacientes portadores de coronariopatias (23) tiveram um nível médio de hemoglobina pré transfusional de $8 \pm 1,3 \mathrm{~g} / \mathrm{dL}$.

$\mathrm{O}$ valor da $\mathrm{Hb}$ pré-transfusional foi menor do que o relatado em outros estudos. Um estudo prospectivo (CRIT) realizado em 284 UTI americanas, no período de 2000 a 2001 , relatou que $44 \%$ dos pacientes admitidos foram transfundidos e que o valor da $\mathrm{Hb}$ pré-transfusional foi 8,6 g/dL ${ }^{16}$. Em estudo realizado em paises europeus (ABC), em 1999, observaram-se taxas de $29 \%$ de anemia na admissão da UTI, 37\% de pacientes transfundidos e um valor "gatilho" de $\mathrm{Hb}$ de $8,4 \mathrm{~g} / \mathrm{dL}^{7}$. Este estudo foi realizado em um período de 4 semanas e 3534 pacientes de 146 hospitais foram avaliados. No Reino Unido $53 \%$ dos pacientes foram transfundidos e o nível de $\mathrm{Hb}$ pré-transfusional foi de $9 \mathrm{~g} / \mathrm{dL}^{23}$. Um estudo canadense avaliou 5000 pacientes e $31 \%$ receberam transfusão de hemácias $^{24}$. O menor limiar transfusional observado neste estudo em comparação aos estudos citados, deve ter sido determinado pelo maior tempo transcorrido entre o estudo atual e o estudo TRICC, permitindo maior disseminação do conhecimento da segurança do uso de menores limiares transfusionais e de seu uso na pratica diária ${ }^{25}$.

As causas mais freqüentes de transfusão de hemácias nesta casuística foram a presença de anemia (53\%) e sangramento ativo (26,6\%). Estas mesmas indicações foram as mais prevalentes no Reino Unido ${ }^{23}$. A presença de hemorragia e o aumento da oferta tecidual de oxigênio foram as principais causas no estudo canadense. No Canadá, $40 \%$ dos médicos transfundem na presença de um nível de hemoglobina inferior a 10 $\mathrm{g} / \mathrm{dL}^{25}$. Em outro relato, não havia indicação em $29 \%$ dos casos de transfusão de hemácias ${ }^{19}$.

As complicações foram mais freqüentes nos pacientes transfundidos do que nos pacientes não transfundidos. Somente uma reação adversa relacionada à $\mathrm{TH}$ foi relatada em um paciente $(0,28 \%)$, que apresentou uma reação febril e urticariforme. Complicações infecciosas e não infecciosas são descritas em aproximadamente $20 \%$ das transfusões sangüíneas ${ }^{10}$. As práticas transfusionais e os riscos da manutenção de níveis mais baixos de hemoglobina em pacientes gravemente enfermos vêm sendo amplamente questionados nos últimos anos, em razão do risco de transmissão de agentes como os vírus da hepatite $\mathrm{B}$ e $\mathrm{C}$, HIV, citomegalovírus e HTLV, ou de doença de Chagas e sífilis, além do significativo aumento do risco de adquirir infecções nosocomiais ${ }^{10}$.

Em um grupo heterogêneo de 1717 pacientes graves a taxa de infecção foi de $15,4 \%$ em pacientes transfundidos e de $2,92 \%$ no grupo não transfundidos ${ }^{13}$. Os pacientes transfundidos apresentavam aumento seis vezes maior no risco de desenvolver uma infecção enquanto internados. Neste estudo quanto maior foi o numero de unidades transfundidas, maior foi o risco de infecção. As taxas de mortalidade foram $24,3 \%$ em pacientes transfundidos e $10,2 \%$ nos não transfundidos ${ }^{13}$. Em 1136 pacientes avaliados em 145 UTI européias as taxas de mortalidade foram de $23 \%$ em pacientes transfundidos e $17 \%$ em pacientes não transfundidos ${ }^{7}$.

Nos pacientes admitidos para cuidados no pós-operatório foram utilizadas $25 \%$ do total de unidades transfundidas. É muito provável que determinados grupos de pacientes sejam mais freqüentemente transfundidos, como o de pacientes submetidos a procedimentos cirúrgicos de grande porte, portadores de neoplasia e politraumatizados ${ }^{7}$. Em um estudo de pacientes submetidos à intervenção cirúrgica para ressecção de neoplasia, os pacientes não sobreviventes tinham uma taxa de hemoglobina me- 
nor no pré-operatório e foram mais freqüentemente transfundidos do que os sobreviventes ${ }^{26}$. Um total de $77 \%$ dos pacientes politraumatizados recebeu TH no presente estudo. Em um grupo de pacientes politraumatizados, um total $55,4 \%$ foi transfundido, com uma média de 5,8 unidades de concentrado de hemácias ${ }^{27}$.

Algumas limitações dos estudos prospectivos de curta duração devem ser discutidas. A avaliação realizada em curto período de tempo pode influenciar nos resultados por não permitir a percepção do efeito das variações sazonais nas causas de internação ou ainda pela possibilidade de influenciar nas decisões médicas. A única orientação que foi transmitida aos centros participantes é de que nenhuma modificação poderia ser feita nas práticas transfusionais da UTI neste período. Contudo, as decisões sobre o metodo de coleta de dados, se "em tempo" ou de forma retrospectiva no final de duas semanas, ou ainda, por quem os dados deveriam ser colhidos, ficaram a cargo do investigador principal. Por outro lado, estudos por tempos mais prolongados podem apresentar uma grande taxa de desistências ou de pacientes não incluídos, dificuldades estas, mais difíceis de serem contornadas. Da mesma maneira as análises retrospectivas, apesar de não influenciarem nos resultados, determinam um grande número de dados perdidos. Pode-se questionar ainda se a população do estudo representa verdadeiramente a realidade das UTI brasileiras. Contudo considerou-se a amostra representativa uma vez que o estudo incluiu UTI de 6 estados brasileiros (1 na região norte, 2 na região sul, 2 na região sudeste e 1 na região centro-oeste) com uma distribuição similar entre instituições públicas e privadas. Até o momento este é o primeiro estudo multicêntrico que avaliou a prevalência de anemia e a prática de transfusão de hemácias em UTI brasileiras.

Os dados do presente estudo mostraram um limiar de hemoglobina para a transfusão de hemácias de 7,7 $\pm 1,1 \mathrm{~g} / \mathrm{dL}$, o que é ligeiramente inferior ao relatado em outros estudos. Apesar de valores similares de APACHE II na admissão, os pacientes transfundidos tiveram maior taxa de mortalidade, embora sem significado estatístico. Os pacientes transfundidos também ficaram mais tempo em ventilação mecânica e necessitaram de fármacos vasoativos com maior freqüência. Contudo, tinham mais disfunções orgânicas na admissão, o que sugeria maior gravidade.
Hospitais Participantes:

Hospital São Lucas e Hospital Cardiotrauma do Rio de Janeiro - Dr. Marcos Freitas Knibel; Hospital de Base de São José do Rio Preto - Suzana M Lobo; Hospital da Universidade Estadual de Londrina - Dra. Cintia Magalhães Carvalho Grion; Hospital de Clínicas de Porto Alegre - Dr Gilberto Friedman; Hospital Padre Albino e Hospital Emilio Carlos de Catanduva - Dr Jorge Luis Valiatti; Escola Paulista de Medicina - Dra Flávia Ribeiro Machado; Santa Casa de Misericórdia de São Paulo e Santa Isabel - Dr. Paulo Antonio Chiavone; Hospital Unimed de Limeira - Dr. Luis Eduardo Miranda Paciência; Hospital Regional de Assis - Dr Juarez de Paula; Beneficência Portuguesa de S J Rio Preto - Dr Sérgio Mussi Guimarães; Hospital Pró-Cardíaco - Rio de Janeiro - Dr Rubens Carmo Costa Filho , Dr. João Luiz Ferreira Costa; Hospital Santa Helena de Goiânia - Dra. Gleida Alves Borges; Hospital Evangélico de Goiânia - Dr Hemerson Casado Garcia e Dr Marcellus Gazola Grilo; Universidade Federal do Rio Grande do Norte (UFRN) - Dr. Kerginaldo Paulo Torres; Hospital Novo Atibaia de Atibaia - Dr. Rubens Sérgio da Silva Franco

\section{REFERÊNCIAS}

01. Lobo SM - Anemia e transfusão de hemáceas em pacientes críticos. RBTI; 2004;16:7-8.

02. Vincent $\mathrm{JL}$, Sakr Y, Creteur $\mathrm{J}$ - Anemia in the intensive care unit. Can $\mathrm{J}$ Anesth, 2003; 50:(Suppl6):S53-S59.

03. Nguyen BA, Bota DP, Melot $\mathrm{C}$ et al - Time course of hemoglobin concentrations in nonbleeding intensive care unit patients. Crit Care Med, 2003; 31:406-410.

04. Faquin WC, Schneider TJ, Goldberg MA - Effect of inflammatory cytokines on hypoxia-induced erythropoietin production. Blood, 1992; 79:1887-1994.

05. Spahn D, Marcucci C - Blood management in intensive care medicine: CRIT and ABC--what can we learn? Crit Care, 2004; 8:89-90.

06. Napolitano LM - Scope of the problem: epidemiology of anemia and use of blood transfusions in critical care. Crit Care, 2004;4:(Suppl2): S1-S8.

07. Vincent JL, Baron JF, Reinhart $\mathrm{K}$ et al - Anemia and blood transfusion in critically ill patients. JAMA, 2002;288:1499-1507.

08. Fernandes CJ Jr, Akamine N, De Marco FV et al - Red blood cell transfusion does not increase oxygen consumption in critically ill septic patients. Crit Care, 2001; 5:362-367.

09. Walker RH - Transfusions risks. Am J Clin Pathol, 1987; 88:374-378.

10. Goodnough LT - Risks of blood transfusion. Crit Care Med, 2003; 31 : S678-S686.

11. Shorr AF, Jackson WL, Kelly KM et al - Transfusion practice and blood stream infections in critically ill patients. Chest, 2005; 127:1722-1728.

12. Taylor RW, Manganaro L, O'Brien J et al - Impact of allogenic packed red blood cell transfusion on nosocomial infection rates in the critically ill patient. Crit Care Med, 2002;30:2249-2254.

13. Vamvakas EC, Blajchman MA - Deleterious clinical effects of transfusion-associated immunomodulation: fact or fiction? Blood, 2001; 97:1180-1195.

14. Weiskopf RB - Do we know when to transfuse red cells to treat acute anemia? Transfusion, 1998;38:517-521.

15. Hebert PC, Wells G, Blajchman MA et al - A multicenter, randomized, 


\section{ANEMIA E TRANSFUSÕES DE CONCENTRADOS DE HEMÁCIAS EM PACIENTES GRAVES NAS UTI BRASILEIRAS* (PELO FUNDO-AMIB)}

controlled clinical trial of transfusion requirements in critical care. $\mathrm{N}$ Engl J Med, 1999; 340:409-417.

16. Corwin HL, Gettinger A, Pearl RG et al - The CRIT Study: Anemia and blood transfusion in the critically ill - current clinical practice in the United States. Crit Care Med, 2004; 32:39-52

17. Wu WC, Rathore SS, Wang Y et al - Blood transfusion in elderly patients with acute myocardial infarction. N Engl J Med, 2001; 345:1230-1236.

18. Yang X, Alexander KP, Chen AY et al - The implications of blood transfusions for patients with non-ST-segment elevation acute coronary syndromes: results from the CRUSADE National Quality Improvement Initiative. J Am Coll Cardiol, 2005; 46:1490-1495.

19. Corwin HL, Parsonnet KC, Gettinger A - RBC transfusion in the ICU. Is there a reason? Chest, 1995; 108:767-771.

20. Littenberg B, Corwin H, Gettinger A et al - A practice guideline and decision aid for blood transfusion. Immunohematol, 1995; 11:88-94.

21. American College of Chest physicians / Society of Critical Care Medicine Consensus Conference: definitions for sepsis and guidelines of innovative therapies in sepsis. Crit Care Med, 1992;20:864-874.

22. Vincent JL, de Mendonça A, Cantraine F et al - Use of the SOFA score to assess the incidence of organ dysfunction/failure in intensive care units: results of a multicentric, prospective study. Crit Care Med, 1998;26:1793-1800.

23. Rao MP, Boralessa $\mathrm{H}$, Morgan $\mathrm{C}$ et al - Blood component use in critically ill patients. Anaesthesia, 2002; 57:530-534.

24. Hebert PC, Wells G, Tweeddale $M$ et al - Does transfusion practice affect mortality in critically ill patients? Transfusion Requirements in Critical Care (TRICC) Investigators and the Canadian Critical Care Trials Group. Am J Respir Crit Care Med, 1997; 155:1618-1623.

25. Hebert PC - Transfusion requirements in critical care (TRICC): a multicentre, randomized, controlled clinical study. Transfusion Requirements in Critical Care Investigators and the Canadian Critical care Trials Group. Br J Anaesth, 1998; 81: (Suppl1):25-33.

26. Magno LA, Rezende E, Assunção $M$ et al - Transfusão sangüínea pósoperatória em pacientes submetidos à cirurgia eletiva para retirada de neoplasia gastrintestinal. RBTI, 2004;16:9-13.

27. Shapiro MJ, Gettinger A, Corwin HL et al - Anemia and blood transfusion in trauma patients admitted to the intensive care unit. J Trauma, 2003;55:269-274. 